Max-Planck-Institut für demografische Forschung

Max Planck Institute for Demographic Research

Konrad-Zuse-Strasse 1 - D-18057 Rostock - GERMANY

Tel +49 (0) 3812081 - 0; Fax +49 (0) 3812081 - 202;

http://www.demogr.mpg.de

MPIDR WORKING PAPER WP 2015-008

NOVEMBER 2015 (REVISED OCTOBER 2016)

\title{
A unified framework of demographic time
}

\author{
Tim Riffe (riffe@demogr.mpg.de) \\ Jonas Schöley \\ Francisco Villavicencio
}

This working paper has been approved for release by: Mikko Myrskylä (sekmyrskyla@demogr.mpg.de), Head of the Laboratory of Population Health and Head of the Laboratory of Fertility and Well-Being.

(C) Copyright is held by the authors.

Working papers of the Max Planck Institute for Demographic Research receive only limited review. Views or opinions expressed in working papers are attributable to the authors and do not necessarily reflect those of the Institute. 


\section{A unified framework of demographic time}

Tim Riffe*1, Jonas Schöley ${ }^{2,3}$, and Francisco Villavicencio ${ }^{2,3}$

${ }^{1}$ Max Planck Institute for Demographic Research

${ }^{2}$ University of Southern Denmark

${ }^{3}$ Max-Planck Odense Center on the Biodemography of Aging

October 4, 2016

*riffe@demogr.mpg.de 


\begin{abstract}
Demographic thought and practice is largely conditioned by the Lexis diagram, a two-dimensional graphical representation of the identity between age, period, and birth cohort. This relationship does not account for remaining years of life or other related time measures, whose use in demographic research is both underrepresented and incompletely situated. We describe a three-dimensional relationship between six different measures of demographic time: chronological age, time to death, lifespan, time of birth, time of death, and period. We describe four identities among subsets of these six measures, and a full identity that relates the six of them. One of these identities is the age-period-cohort identity, while the others are relatively novel. We provide a topological overview of the diagrams that pertain to these identities. The 3-d geometric representation of the full six-way identity is proposed as a coordinate system that fully describes temporal variation in demographic data. We offer this framework as an instrument to enable the discovery of yet-undescribed relationships and patterns in formal and empirical demography.
\end{abstract}

Keywords. Age structure, formal demography, data visualization, age period cohort.

\title{
1 Introduction
}

In the course of training, all demographers are introduced to the Lexis diagram, a convenient graphical identity between the three main time measures used to structure demographic stocks and flows: Age, period, and birth cohort. This popular representation does not account for remaining years of life and other related time indices that may be of interest to researchers and policy makers.

We wish to draw attention to three time indices that are complementary to age (A), period (P) and birth cohort (C). The first such index is time-to-death, which we refer to as "thanatological age" (T) in contrast to "chronological age" (A). The second index is death cohort (D), which groups all individuals (of different ages) dying in the same time period. Finally, lifespan (L) or age-at-death itself is an index by which data may be 
structured. We therefore have six time measures in total to relate. We call these measures of demographic time because each, except period, depends on the timing of birth, death, or both.

The Lexis diagram can be understood as an APC plane that relates age, period, and birth cohort. Other such planes are also identifiable. The "thanatological" counterpart to APC is an identity between thanatological age, period, and death cohort, TPD. A third identity relates thanatological age, chronological age, and lifespan, TAL. A fourth identity relates lifespan, birth cohort, and death cohort, LCD. We call three-way identities of this sort "triad identities".

Each of these four triad identities (APC, TPD, TAL, and LCD) is sufficiently described by any two of its constituent indices, making the third index redundant. For instance, if the exact age of an individual at a particular time is known, the birth cohort to which he or she belongs can be immediately derived. Each of these four identities also lacks a major dimension of time. The TAL identity lacks calendar time, the LCD identity is ageless, APC lacks an endpoint in time, and TPD lacks a starting point in time. To our knowledge, the only triad identity that has received serious treatment at the time of this writing is the APC identity. Different aspects of the APC identity have been discussed since at least 1868 (Knapp 1868), and discussion remains lively today. Here we relate the six major indices of time in a geometric identity, in much the same spirit as the work on APC relationships done between the late 1860s and mid 1880s. ${ }^{1}$

Our goal is to describe the geometric identity between all six measures of demographic time, a hexad identity, that may be useful or an intuitive referent for demographers in the same way as the Lexis diagram. At the same time, this identity relates the four triad identities we have mentioned. We give a bottom-up description of how the six dimensions of time relate in a single framework, building from familiar components to the full relationship. A similar six-way identity was in fact originally described by Lexis (1875) for the case of marriage cohorts. The framework we describe is more general and adaptable for such event history scenarios. We begin by defining some terms used

\footnotetext{
${ }^{1}$ See e.g., Keiding (2011) for an overview of that literature.
} 
throughout the manuscript. We then explore all combinations of two time measures, the dyadic relationships, followed by the four triad identities, and finally the hexad identity. We give a systematic topological overview of the different elements of demographic time.

Just as the Lexis diagram has been a fundamental instrument to teach demography for decades, we hope that the demographic time measures and their graphical depictions presented here will be helpful to teachers and young demographers. The temporal relationships we describe will also be useful for researchers to better detect and understand patterns data, and for methodologists to better account for the structure of data in demographic methods.

\section{Definitions}

\subsection{Technical terminology}

In describing this relationship we attempt to adhere to a rigorous terminology. The following list describes some of the more important terms we use.

Demographic time measures are any of the six time indices discussed to describe demographic time: chronological age $(\mathrm{A})$, period $(\mathrm{P})$, birth cohort $(\mathrm{C})$, thanatological age $(\mathrm{T})$, lifespan $(\mathrm{L})$, and death cohort (D).

Dyads, triads, and hexads are any set of two, three, or six unique time measures, respectively.

A triad identity is a triad with the property that each of its members can be derived from the other two with no additional information. There are four triad identities: APC, TPD, TAL, and LCD.

A temporal plane is any $(x, y)$-mapping of a dyad of time measures.

Using this terminology, we say that the "Lexis" measures constitute a triad identity between chronological age, period, and birth cohort. Each dyad combination of elements in this identity can be mapped to a temporal plane, the Lexis diagram. If we know that 
Mindel turned 50 on the 21st of May, 1963, then we also can derive that she was born on the 21st of May, 1913. Hence, any two pieces of information in this case will give the third, and the same holds for the other triad identities.

\subsection{Time measures}

We describe time in terms of years, the dominant time scale for human demography, although all relationships are scalable to any time unit. We therefore speak of calendar time. We also describe the framework in terms of human lifespans, although it applies in a more general sense to any durations observed over time. This is to say, birth may be translated to entry, and death to exit, or any other terminal state. The six measures of time we consider are defined in Table 1, both in the demographic sense we describe, as well as in a more general event history interpretation.

Table 1: Definitions of the six time measures.

\begin{tabular}{lcll}
\hline Time measure & Short & Demographic def. & Event history def. \\
\hline chronological age & A & Time since birth & Time since start of exposure \\
period & P & calendar time & calendar time \\
birth cohort & C & calendar time of birth & calendar time of exposure start \\
thanatological age & T & time until death & time until event \\
death cohort & D & calendar time of death & calendar time of event \\
lifespan & L & duration of life & duration of exposure
\end{tabular}

The concepts of thanatological age and death cohorts are likely less familiar to readers than the other measures we consider. Thanatological age is sometimes referred to as remaining years of life, time-to-death, years left (Vaupel 2009, Villavicencio \& Riffe 2016), prospective age (Sanderson \& Scherbov 2007), or residual life, but we prefer the term thanatological age (Riffe 2015). Age in this general sense marks a position on a lifeline with respect to one of its endpoints. Chronological age and thanatological age are in this way complementary, duals. Thanatological age is meaningful without much justification: It is the measure we all want to know, the thing we approximate with remaining life expectancy.

Cohorts in general associate individuals that share a characteristic. In demography the grouping characteristic is often a combination of place and time, such as a cohort 
of young demographers passing through a particular graduate program. In this instance already, we accommodate the notion of a cohort for both the start and endpoints of the program, saying for example, "the class of 2015" instead of the "graduating cohort of 2015 ", in contrast to "cohort 37", the 37th class of entering students since the start of the program. These concepts are analogous to the ideas of birth and death cohorts we use here, but we do not often refer to the deaths of a given year as a death cohort. In the time preceding death, the members of a given death cohort have much in common, despite heterogeneity with respect to time of birth. ${ }^{2}$ If the reader accepts this premise, then the abstract construct of a death cohort is also meaningful in the way that other cohort measures are. In event history or non-human contexts, anologs to death cohorts in this framework may be even more meaningful.

Much of the work of demography is directed at the study of lifespan. Lifespan is synonymous both with longevity, chronological age at death, and thanatological age at birth. One's ultimate completed lifespan is constant throughout life, though we have no knowledge of it until death: It is assigned retrospectively. Demographers have more often used lifespan or age-at-death as a measure of mortality, or similar, than as a measure on which to compare individuals or structure data.

Treating lifespan, death cohorts, and thanatological age as temporal structuring variables enables new classes of comparisons, models of understanding, and discovery, akin to those unlocked by breaking down demographic phenomena by chronological age, period, and birth cohort. The following sections, in this sense, provide an exhaustive classification of the ways in which these six measures of time can be juxtaposed to such ends.

\section{From dyads to the triad identities}

We distinguish between two kinds of dyads: informative dyads and uninformative dyads. Informative dyads are any pair of measures from which a third time measure can be derived, forming a triad identity. There are $15=\left(\begin{array}{l}6 \\ 2\end{array}\right)$ possible dyads in our set of time

\footnotetext{
${ }^{2}$ Death cohorts lack a shared identity, so any kind of emergent homogeneity in a death cohort probably has a physiological basis.
} 
measures, 12 of which are informative, and three of which have no derived time measure, and are therefore called uninformative. For instance, if we take the dyad TA, L is the derived measure, and TAL the corresponding triad identity. In contrast, nothing can be derived from the LP dyad: You can have an eventual lifespan of 100 in the year 2016 and still be alive with the same eventual lifespan in 2017.

In this section we systematically map each dyad to its temporal plane, and we synthesize these into the four primary identities and their essential diagrams. We first discuss the choice between mapping dyads to Cartesian coordinates or to isotropic coordinates, which constrain the scales of all measures to be equal. We then systematically render the 15 dyad-based diagrams that can be derived from the six time measures. Of these 15,12 diagrams can be distilled into just four, the triad identity diagrams. Each triad identity diagram is then briefly discussed with suggested or speculated applications.

\subsection{The question of mapping}

Any mapping of two time measures to an $(x, y)$ coordinate system constitutes a temporal plane. If the two given time measures are members of the same triad identity, the third member is a derived measure. If we assign $\mathrm{A}$ to $y$ and $\mathrm{P}$ to $x$, thereby implying $\mathrm{C}$ (and the APC triad identity) we state this relationship explicitly by writing $\operatorname{AP}(C)$. The temporal plane that corresponds to this informative dyad is the contemporary representation of the Lexis diagram (Lexis 1875, Pressat 1961). The informative dyads AC(P) and CP(A) also belong to the Lexis identity, but imply different less-common rotations and projections of the Lexis diagram. ${ }^{3}$

For each dyad there is a fundamental question of how to map the constituent coordinates to a Cartesian temporal plane. Typically one forces parity between time units within a specified dyad, mapping one element directly to $x$ and the second element directly to $y$, resulting in a $90^{\circ}$ angle between the $x$ and $y$ axes. In this case it is conventional

\footnotetext{
${ }^{3}$ While uncommon as diagram orientations, these latter two dyads are used to tabulate data for different kinds of rates and probabilities. Measures based on the AC dydad are variously referred to as Type III rates (Caselli et al. 2006) or vertical parallelograms (Wilmoth et al. 2007). The CP dyad delineates Type II rates (Caselli et al. 2006), a.k.a. horizontal parallelograms, for instance used to calculate cohort lifetables in the Human Mortality Database (Wilmoth et al. 2007).
} 
to force a unity aspect ratio between the $x$ and $y$ axes, such that the derived measure, if any, is then accidentally present in a $45^{\circ}$ ascending or descending angle, depending on the dyad and axis orientation.

It has long been noted (Perozzo 1880, Zeuner 1869) that the derived time measure (usually birth cohort) is longer than either the age or period axes when plotted at $45^{\circ}$. If a right angle and unity aspect ratio is forced between the dyad, the derived measure is always stretched by $\sqrt{2}$, or $41 \%$. In the case of informative dyads, another logical mapping is to translate to $(x, y)$ coordinates that force $60^{\circ}$ angles between the three measures. Such a mapping ensures that the spatial units are equal for the three measures, and we therefore refer to it as the isotropic mapping. The isotropic mapping is comparable to using ternary or barycentric coordinate systems. Under the isotropic representation, the three variants of each triad identity are simple rotations of one another, and they require no rescaling.

\subsection{Dyads to diagrams}

Each of the 15 dyads, an explanation or simple example, and the corresponding diagram representations are summarized in Table 2. Each informative dyad is a subset consisting of two elements from one of the four triad identities (APC, TPD, TAL, LCD), which we analyze in detail in further sections. The uninformative dyads are simply pairs of time measures that do not have a derived measure, and therefore are not contained in any of these four triad identities. 
Table 2: All dyadic juxtapositions of the six measures of demographic time.

Note: The temporal planes are named after the two given time scales. The derived scale is appended in parentheses. Contrary to mathematical convention we name the ordinate scale first and the abscissa scale second. This is to be consistent with the established $A P C$ and $A C P$ terms.

\begin{tabular}{|c|c|c|c|}
\hline Relationship & Description & Cartesian & Isotropic \\
\hline \multicolumn{4}{|c|}{ VARIANTS OF APC } \\
\hline
\end{tabular}

$\begin{array}{ll}A P(C) & \text { The } \mathrm{AP}(\mathrm{C}) \text { temporal plane } \\ C=P-A & \begin{array}{l}\text { constitutes the classical Lexis } \\ \text { diagram. }\end{array}\end{array}$

The $\mathrm{AC}(\mathrm{P})$ temporal plane is $A C(P) \quad$ equivalent to the Lexis diagram $P=C+A$ except birth cohort is given and period is derived rather than the other way around.

The $\mathrm{CP}(\mathrm{A})$ temporal plane is $\begin{array}{ll}C P(A) & \begin{array}{l}\text { equivalent to the Lexis diagram } \\ \text { except birth cohorts are given }\end{array} \\ A=P-C & \begin{array}{l}\text { and age is derived rather than } \\ \text { the other way around. }\end{array}\end{array}$

\section{VARIANTS OF TPD}
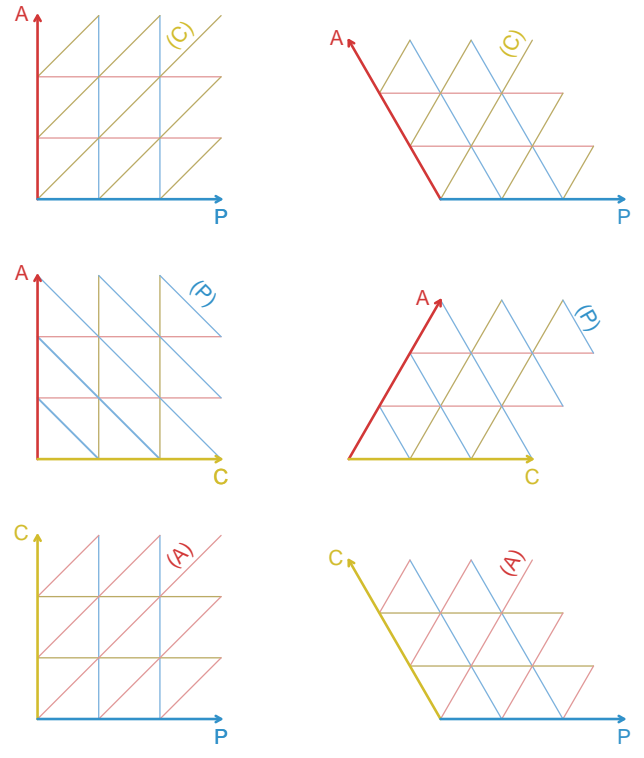

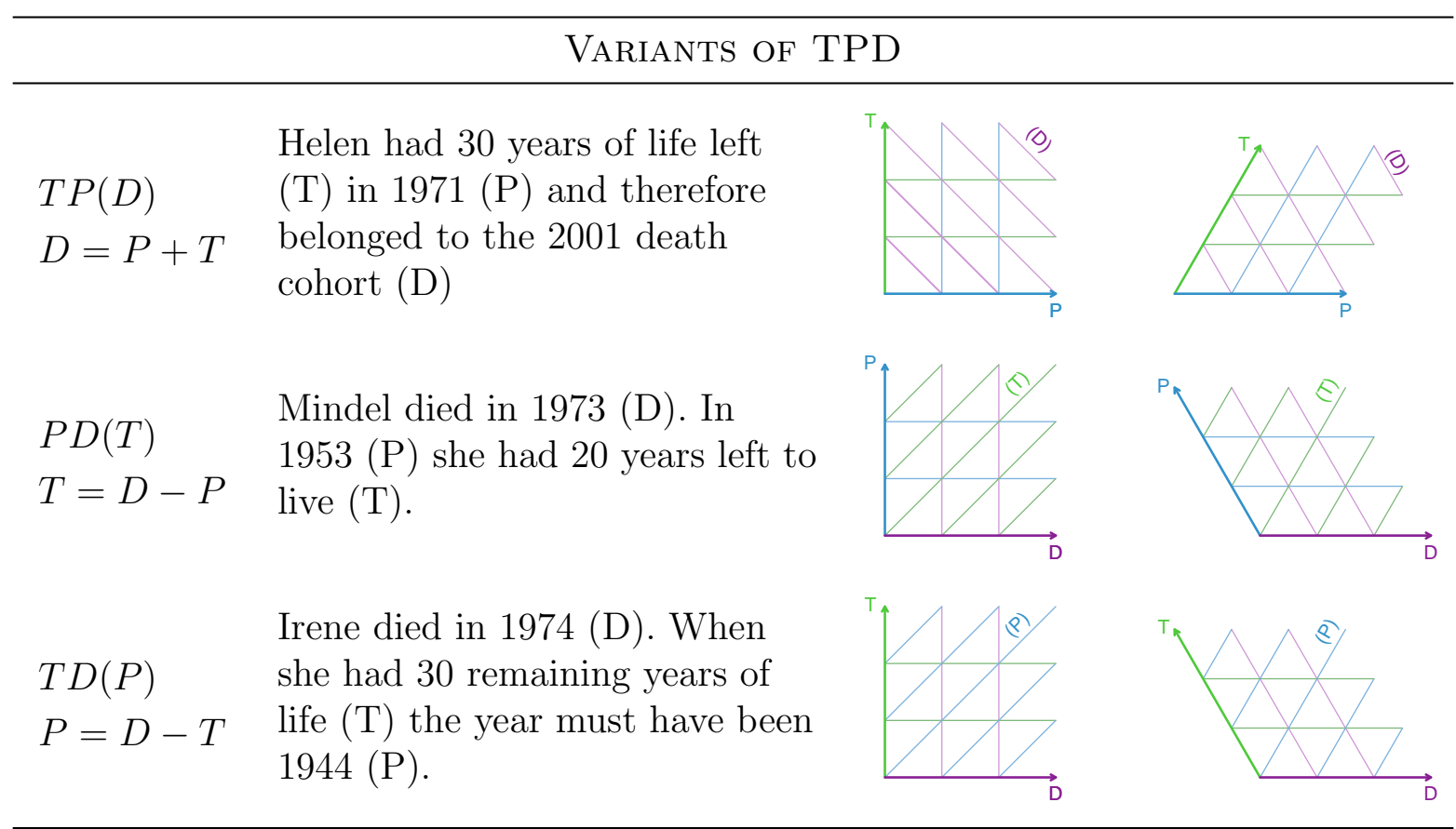

VARIANTS OF TAL 
$T A(L) \quad$ The time already lived and the $L=T+A \quad$ total lifespan.
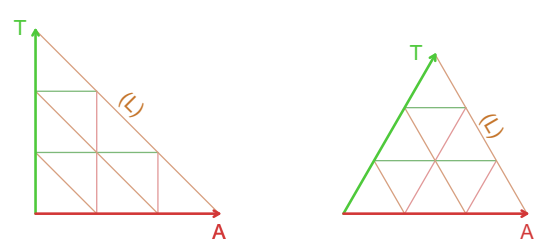

$T L(A)$

Helen lived to the age of $86(\mathrm{~L})$. $A=L-T$ When she had 20 years left (T) she must have been $66(\mathrm{~A})$.
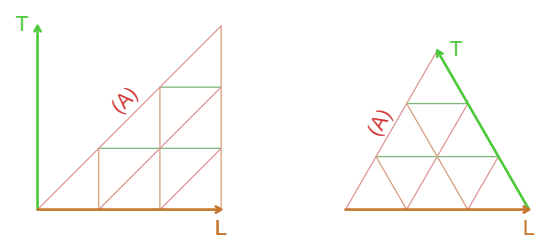

Tim is 34 years old (A) and will $\begin{array}{ll}A L(T) & \text { live to the age of } 96(\mathrm{~L}), \text { leaving } \\ T=A-L & \text { him } 62 \text { years }(\mathrm{T}) \text { to settle } \\ & \text { affairs. }\end{array}$
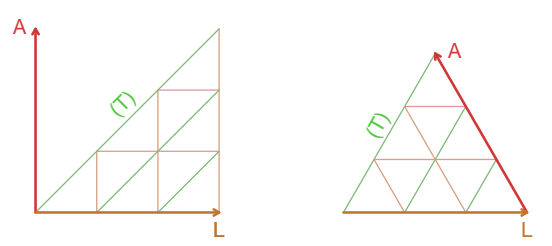

\section{VARIANTS OF LCD}

$\begin{array}{ll}L C(D) & \text { Àngels was born in } 1940(\mathrm{C}) \text { and } \\ D=C+L & \text { she lived to be } 64(\mathrm{~L}), \text { implying } \\ \text { an untimely death in } 2004(\mathrm{D})\end{array}$
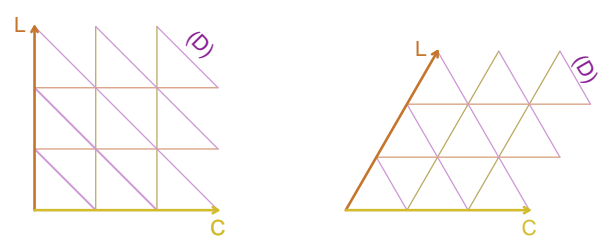
$C D(L)$
$L=D-C$

Pascal was born in 1893 (C) and died in 1964 (D), implying a lifespan of $71(\mathrm{~L})$, or so.
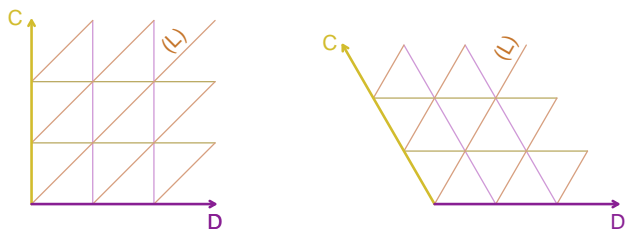

Margaret died in Dec., 1995 (D)
$L D(C) \quad$ with a completed lifespan of 96
$C=D-L \quad(\mathrm{~L})$, putting her birth year in 1900 (C).
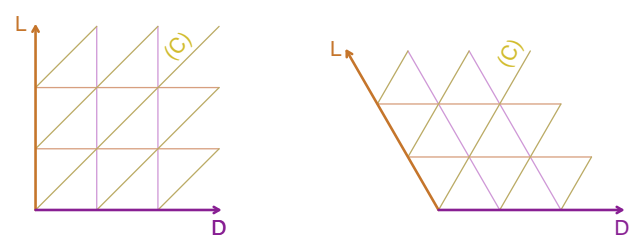

The Uninformative Dyads

The LP plane is

non-informative. No additional

$\mathrm{LP}(-) \quad$ measures can be derived

knowing just lifespan and period.
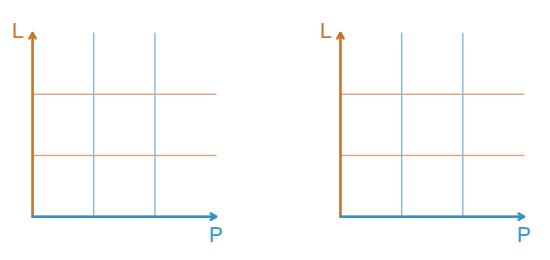

The CT plane is non-informative. No additional CT(-) measures can be derived knowing just birth cohort and thanatological age.
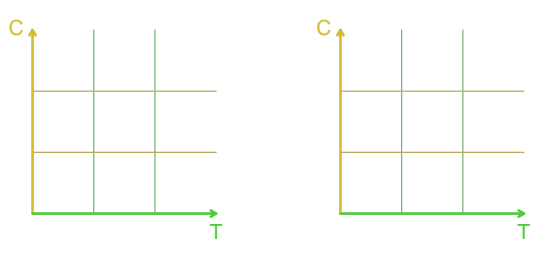
The AD plane is

non-informative. No additional

$\mathrm{AD}(-) \quad$ measures can be derived

knowing just death cohort and

age.
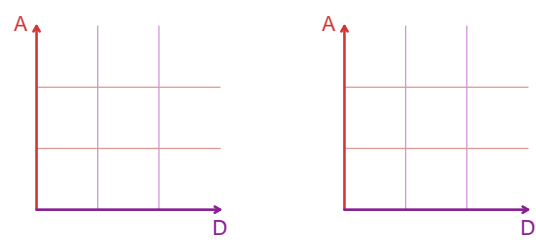

Most of what we know about how rates change over age and time comes from the very first juxtaposition in Table $2, \mathrm{AP}(\mathrm{C})$. While $\mathrm{CP}(\mathrm{A})$ and $\mathrm{AC}(\mathrm{P})$ are statistically redundant when exact times are used, they are not fully redundant if based on discrete double-classification of data, as often provided in aggregated official statistics. Double classified data are found on the APC diagram in the shape of squares (AP), horizontal parallelograms $(\mathrm{AC})$ and vertical paralellograms (PC), and these are commonly used to compute different kinds of demographic rates and probabilities (Caselli et al. 2006, p63). The other dyadic juxtapositions (involving the measures $\mathrm{T}, \mathrm{D}$, or L) can be considered as either rare or novel ways of structuring or viewing temporal variation in demography, and these imply new families of rates and probabilities.

\subsection{The triad identities}

There are $20=\left(\begin{array}{l}6 \\ 3\end{array}\right)$ ways to choose three time indices out of six, of which four form a triad identity: APC, TPD, TAL, and LCD. Given the three time measures from any of the triad identities, one can derive no further time measures. If one selects three random time indices that do not form any of these four triad identities $(20-4=16$ possibilities $)$, this property does not hold. For instance, in the triad APT, age and period are not sufficient to determine thanatological age. Given the triad APT one can however derive the remaining three time measures.

Triad identities are more meaningful than uninformative dyads. This is so even in the absence of data, due to the underlying relationship between measures. Each of the triad identities can accommodate some version of a lifeline, for instance. In the following, we therefore lay out the four primary diagrams that belong to the triad identities. The question of which diagram mapping is relevant to a given demographic phenomena is a function of patterns in the data. The best diagram is the one that captures all meaningful 
variation in the data. If APC highlights meaningful variation in a phenomenon, then its representation as such is useful. The same holds for the other identities. With each diagram in following we comment or speculate on its potential uses.

\subsubsection{APC: Chronological age, period, and birth cohort}

The so-called Lexis diagram has long been used in demography as a conceptual tool for structuring data, observations, and rate estimation, as inspiration for work on statistical identification, and as the coordinate basis of contemporary Lexis-surfaces. ${ }^{4}$ Since the Lexis diagram could have been named for others (Keiding 2011, Vandeschrick 2001), and since we compare with other temporal configurations, let us refer to it as the APC diagram, as seen in Figures $1 \mathrm{a}$ and $1 \mathrm{~b}$.

The APC diagram in Figure 1a represents years lived on the $y$ axis, calendar years on the $x$ axis, and birth cohorts as the right-ascending diagonals. This is the most common of several possible configurations of the APC dimensions. Individual lifelines (black) are aligned in the birth cohort direction, starting with birth (filled circle) at chronological age zero, and death (circled x). Any APC surface can be interpreted along each of these three dimensions of temporal structure.

\footnotetext{
${ }^{4}$ Some prefer the term Lexis surface, while others prefer to call them contour maps, heatmaps, or stereograms.
} 
Figure 1: An APC diagram in two projections.

(a) Cartesian

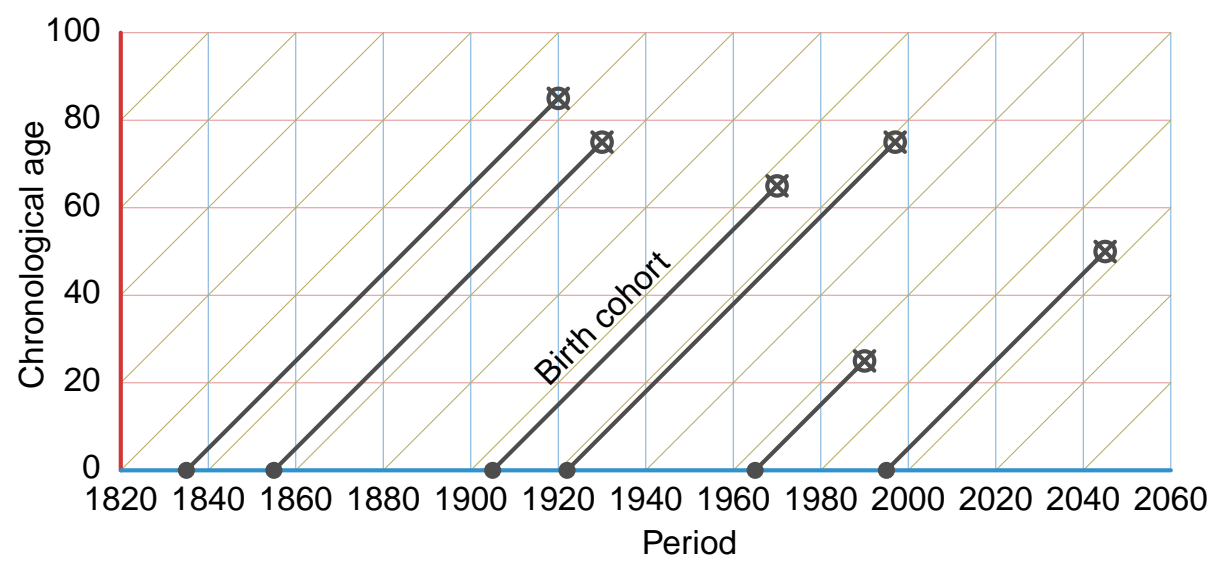

(b) Isotropic

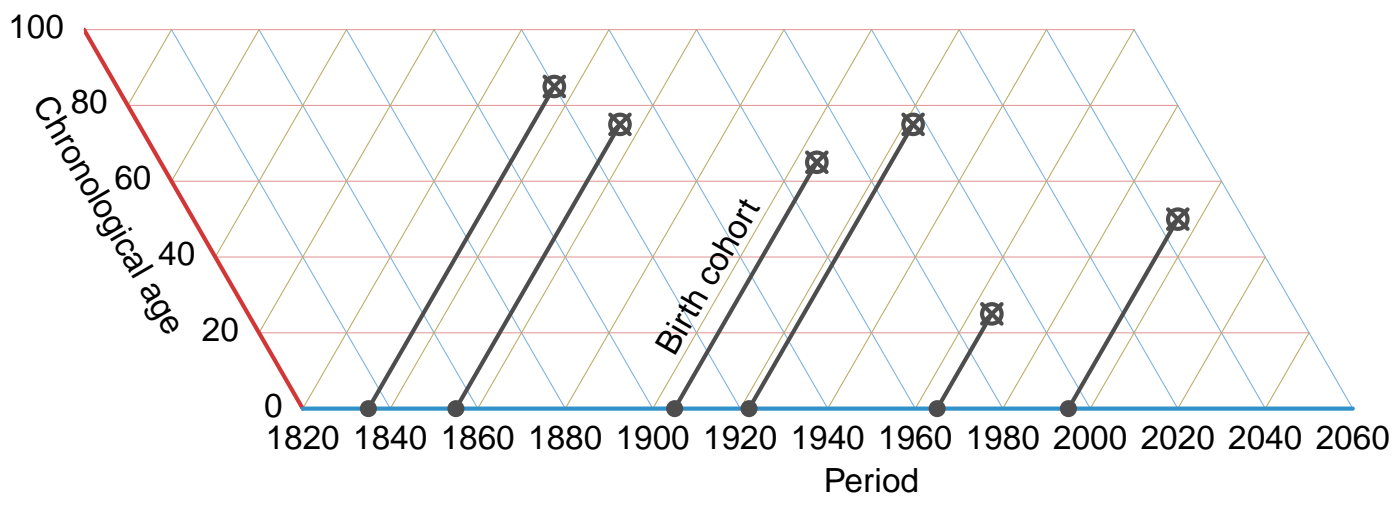

\subsubsection{TPD: thanatological age, period, and death cohort}

The TPD diagram is best imagined as the inverse of the APC diagram. One may take the same individuals represented in Figure 1 and group them by death cohorts (D) instead of birth cohorts (C). Lifelines then descend such that all endpoints align to thanatological age 0 , creating the diagram in Figure 2 in which individuals dying at different ages but in the same time period are grouped together. To our knowledge, the TPD diagram has only appeared once in the literature, as a didactic aid in a proof of symmetry between chronological and thanatological age structure in discrete stationary populations (Villavicencio 
\& Riffe 2016). TPD diagrams may also be useful to arrange events or durations that are logically aligned (or may only be aligned) by time of termination. It may be reasonable to align on termination in cases where this brings preceding patterns of variation into focus.

Figure 2: A TPD diagram in two projections.

(a) Cartesian

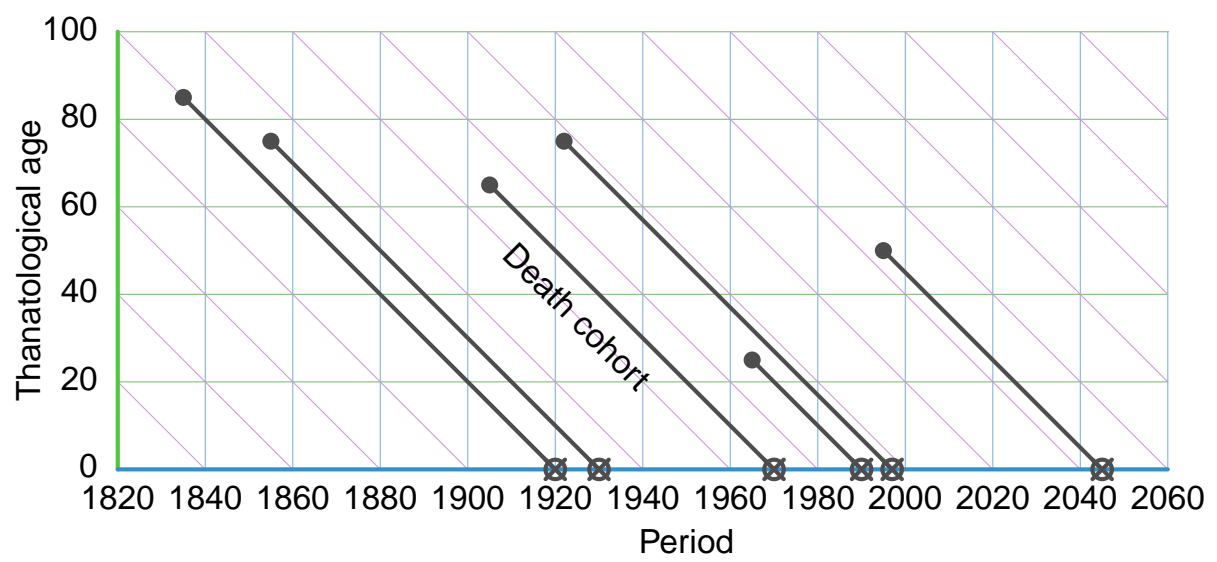

(b) Isotropic

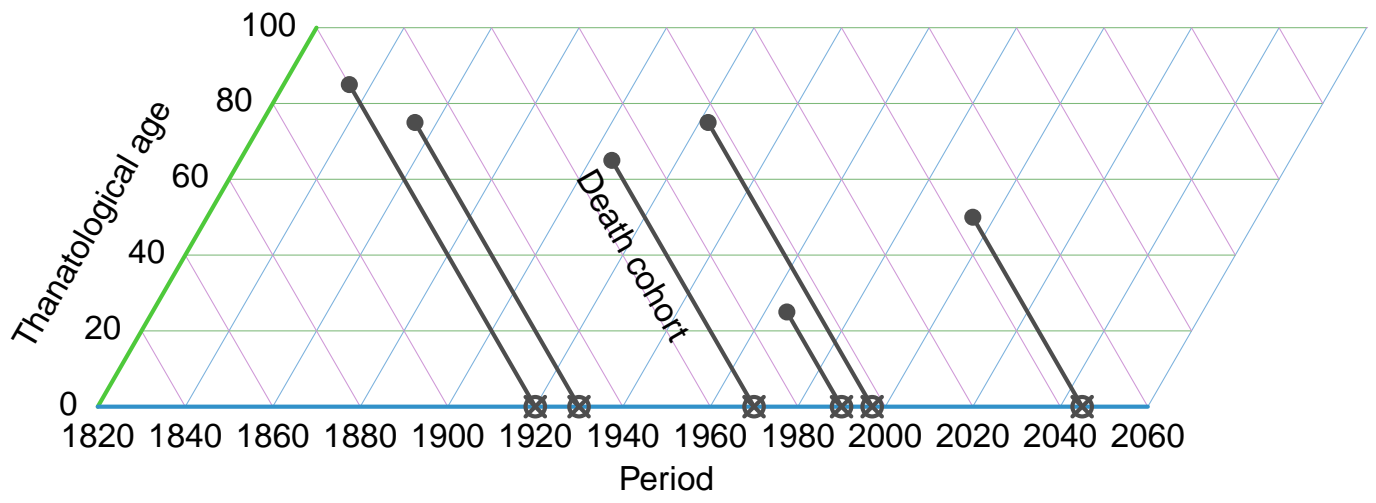

There are several examples of analysis of this kind of data, usually stemming from a lack of information on chronological age. This is the case, for instance, in biodemographic studies in which wild animals with unknown ages are captured and then followed-up until death (Müller et al. 2004; 2007), but it can be generalized to other 
kinds of human and non-human demographic data with a similar structure. For example, in the Barcelona Historical Marriage Database, which collects information about marriage licenses of Barcelona (Spain) from the mid-15th century until the early 20th century, individuals are first identified in their marriage record and then followed-up, but no information is available about dates of birth or age at marriage (Villavicencio et al. 2015). We speculate that TPD diagrams could also be used in biomedical studies for the representation of lifelines preceding deaths from infectious or acquired conditions, when the time of infection or acquisition remains unknown.

\subsubsection{TAL: Thanatological age, chronological age, and lifespan}

TAL is an appropriate diagram to examine processes that vary over the lifecourse. More precisely, the TAL plane can highlight variation that is related to time since birth, time until death, length of life, and their combinations. These key aspects of demographic time are compressed to chronological age only in the APC perspective, which can blend out meaningful variation. Since the lifecourse belongs to the cohort perspective, it is best to think of the TAL plane as belonging to some particular birth cohort. Alternatively, a TAL triangle may be taken as a cross-section through the period dimension, a sort of synthetic TAL plane. To our knowledge, the TAL diagram has only appeared once in the literature, in an exploration and classification of late-life health conditions (Riffe, T. et al. 2015). The TAL diagram in Figure 3 contains no indication of period or cohorts, as calendar time is blended out in this diagram. The lifelines depicted are identical to those shown in APC Figure 1 and TPD Figure $2 .^{5}$ The TAL diagram is useful for characterizing patterns of prevalence of health conditions. We speculate that data structured and aligned in this way may yield hitherto undescribed patterns in other contexts, such as pregnancy (from the perspective of either mother or fetus) by time since conception, time until parturition, and length of gestation, or even smaller event-history time scales, or patterns of growth or reproduction in non-human species.

\footnotetext{
${ }^{5}$ The prior figures contained six lifelines each, but since two of them were of equal length (75), they are overlapped in Figure 3 and appear to be five.
} 
Figure 3: A TAL diagram in two projections.

(a) Cartesian

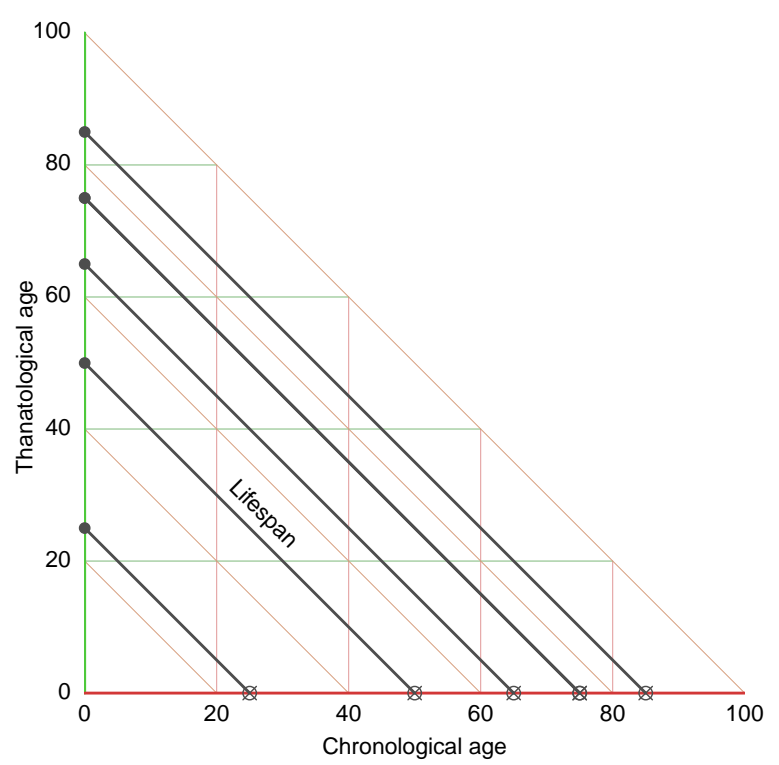

(b) Isotropic

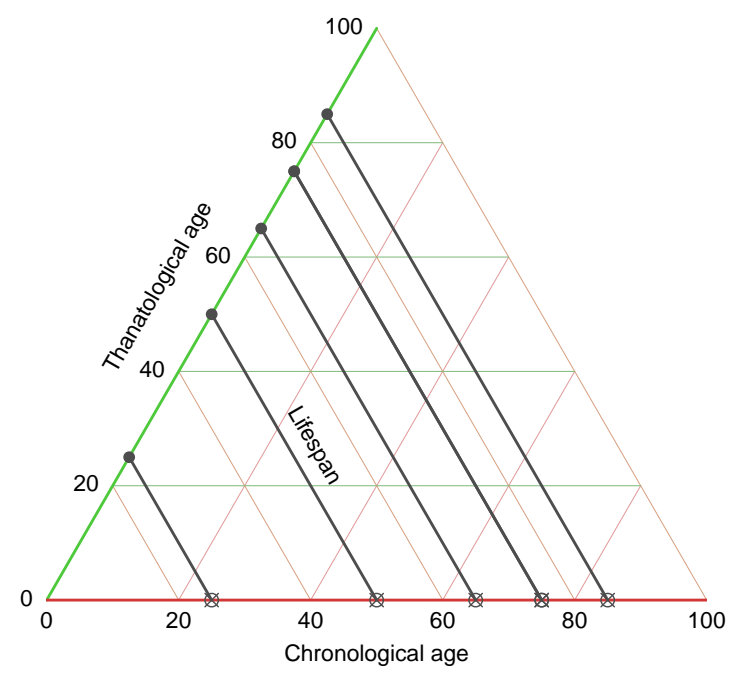

\subsubsection{LCD: Lifespan, birth cohort, and death cohort}

The LCD diagram completes our set of identities. It is based on the relationship between lifespan, birth cohort, and death cohort. In Figure 4a, lifespans are indexed by the $y$-axis, while birth cohorts are indexed by the $x$-axis, and death cohorts are found in descending diagonals. To structure data on these three time measures implies excluding time-varying information over the lifecourse. An individual only ever has one lifespan, one birth cohort, and one death cohort, such that the LCD coordinates of an individual are constant throughout life. The LCD plane is therefore orthogonal to lifelines, and individuals are located with points, rather than life segments. In Figure 4, the same six individuals from previous diagram figures are represented with crossed circles.

We recommend this mapping for plotting surfaces of values that are cumulative or static over the lifecourse. Imagine an LCD surface of cumulative lifecourse consumptive surplus or deficit, or anything else that might vary by lifespan and moment of birth or death, such as children ever born, years of retirement, the size of trees or other aspects of forestry, populations of buildings in large cities, and so forth. Lexis (1875) describes an analogous relationship between marriage cohort, separation cohort, and duration of 
Figure 4: An LCD diagram in two projections.

(a) Cartesian

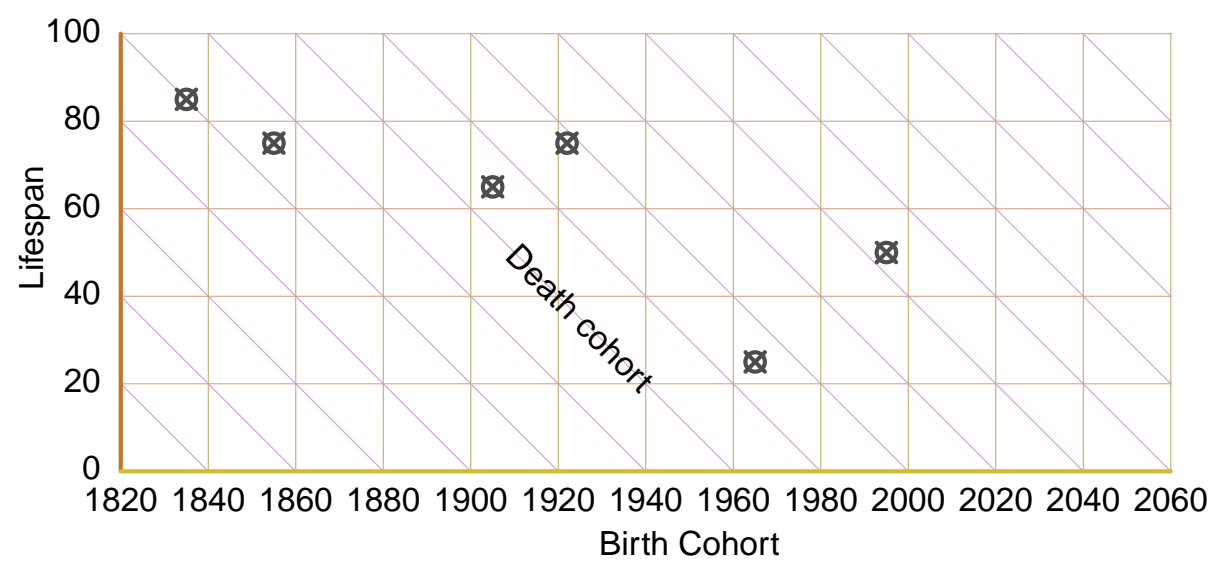

(b) Isotropic

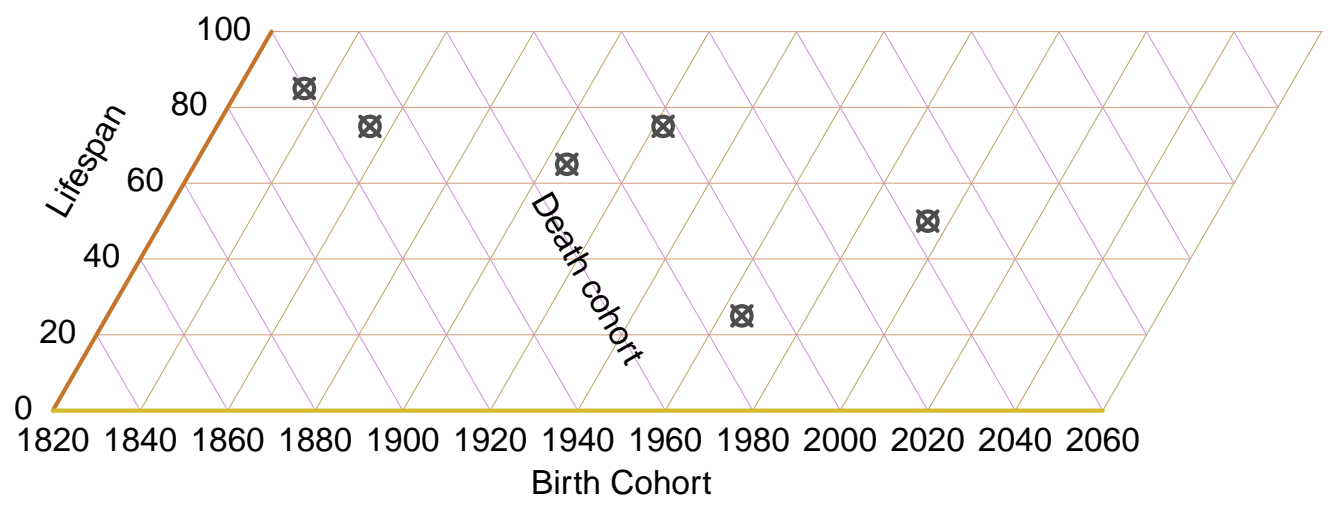

marriage.

\subsubsection{Relationships between the diagrams}

Since demographic time measures themselves depend on the demographic events of fertility and mortality, it stands to reason that fertility and mortality also link the different diagrams. In a loose sense, the LCD diagram has a special dual in the case of an APC surface of mortality. Age at death is equal to lifespan $(A=L)$, period is equal to death cohort $(P=D)$, and birth cohort $(\mathrm{C})$ is just the same in the event of death. These two 
surfaces are exactly equivalent in the case of death counts or densities, since in either case quantities are represented with points, the endpoint of life for which this equivalence holds. For any other demographic measure, however, the LCD diagram is distinct from APC. APC fertility surfaces are in this way different from APC mortality surfaces, as the former represent events or characteristics over the ages in which they occur, allowing for the represention of durations and repetitions. Further, a fertility surface in TPD coordinates again finds an approximate dual in the APC mortality surface. This works because birth marks the starting point of the lifeline which is otherwise missing in TPD coordinates, whereas death marks the endpoint of a lifeline, which is otherwise missing from APC coordinates. This relationship is exact in the special case of a stationary population series. Further reflection may reveal other such dualities.

\section{A tetrahedron relates the six time indices.}

Each of the four triad identities may be thought of as a two-dimensional plane fully defined by any two of its three constituent time indices. In this case, we may imagine any of the excluded time measures as capable of providing depth, a potential $z$-coordinate, for the sake of a mental image. Having a non-redundant third dimension implies a multitude of parallel planes for the given triad identity, each plane belonging to a unique value of the third time dimension. Any of the identities can be extended in this way to fill a space. A space derived by extending any of the triad identities into its lacking dimension implies each of the other triad identities, making a total of six time indices. In essence, the four triad identities may be thought of as the four faces of a tetrahedron. If an additional time measure is added to any face (triad identity), the six demographic time indices can be derived, matching the six edges of the tetrahedron. This three-dimensional construct unifies the six indices of demographic time, and is the subject of this paper.

Let us first more rigorously define the previously-mentioned tetrahedron. Luckily, the edges and vertices of a tetrahedron are easily rendered in a two-dimensional graph, as seen in Figure 5, with vertices labeled in black and the six time indices colored following 
the pattern from Table 2. The tetrahedron is composed with the APC plane at the base and vertex 4 on top. The same graph could be composed in four basic ways, depending on which identity forms the base.

Figure 5: Graph of tetrahedron, with edges labeled by the six demographic time indices. The APC plane is at the base, and vertex four on top.

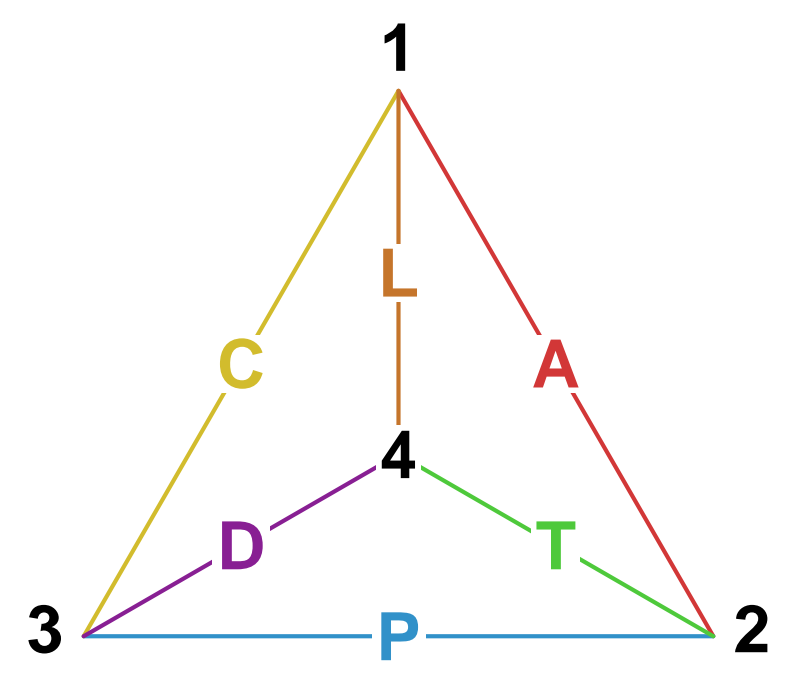

The edges APC at the base define the much-studied APC plane. If the only information we have is chronological age, period, and birth cohort (or just two of these), then we have no access to the vertex 4. Each of the faces of the tetrahedron has this quality. The South face TPD has no access to 1 . The Northeast face, TAL has no connection to 3, and the Northwest face LCD lacks a connection to 2. The four triad identities that make up the faces of the tetrahedron are stuck in "flatland" and do not yield the full 3d space. However, the 16 other possible combinations of three time indices will recreate the full tetrahedron (hexad identity).

\subsection{A geometrical analogy}

A geometrical analogy is pertinent at this point. Any pair of intersecting edges of the tetrahedron may be interpreted as two vectors $\vec{u}$ and $\vec{v}$ that determine a 2-dimensional plane in a Cartesian 3-dimensional space $\left(\mathbb{R}^{3}\right) .{ }^{6}$ Therefore, any third vector $\vec{w}$ of that

\footnotetext{
${ }^{6} \mathrm{~A} 2 \mathrm{~d}$ plane in a 3d space is determined by two linearly independent vectors (with different direction) and a point, but the inclusion of a point is not necessary for the intuitive analogy that we describe here.
} 
plane can be expressed as a linear combination of $\vec{u}$ and $\vec{v}$ (formally, $\vec{w}=\alpha \vec{u}+\beta \vec{v}$ for some $\alpha, \beta \in \mathbb{R}$ ), which is usually described by saying that $\vec{w}$ is linearly dependent on $\vec{u}$ and $\vec{v}$. A similar property can be derived from the information contained in the tetrahedron: $A=P-C$ is a linear combination of $\mathrm{C}$ and $\mathrm{P}$ and it "depends" on them because they all belong to the same APC plane. Analogously, $P=C+A$ "depends" on $\mathrm{C}$ and $\mathrm{A}$, and $C=P-A$ "depends" on $\mathrm{P}$ and A. Given that the faces of the tetrahedron represent the triad identities, any pair of intersecting edges has the same property: The third edge located in the same face of the tetrahedron can be determined by the first two by a simple linear relationship.

Once a $2 \mathrm{~d}$-plane is defined in $\mathbb{R}^{3}$, an additional vector may be sufficient to cover a 3d-space. Nonetheless, this third vector needs to be linearly independent of any pair of vectors of the $2 \mathrm{~d}$ plane - that is, it cannot be expressed as a linear combination of any two vectors on that plane. Again, an analogous property can be observed in the tetrahedron: Say we only have information about the indices of the APC plane; A, $\mathrm{C}$ and $\mathrm{P}$ are not sufficient to determine a thanatological age $\mathrm{T}$, death cohort $\mathrm{D}$, or lifespan L (the three indices that do not belong to the APC plane). So, T, D and L are "independent" of the overall information that can be extracted from the APC plane. However, if two of the three constituent time indices of the APC plane are known (the third one would be unnecessary as it could be derived from the other two), the additional information provided by any of the three "independent" indices T, D or L would be sufficient to cover the whole tetrahedron. For example, suppose we have information about thanatological age $\mathrm{T}$ in addition to $\mathrm{C}$ and $\mathrm{P}$, then $A=P-C, D=P+T$ and $L=T+A=T+P-C=D-C$.

Hence, as with vectors in a $3 \mathrm{~d}$ space, any triad of indices that are independent of each other - that is, none of them can be expressed as the sum or the difference of the other two - generates a full hexad identity or, using an analogous terminology, covers the whole "space" of demographic indices presented here. Graphically, this is equivalent to choosing any combination of three indices that do not belong to the same face of the tetrahedron. 


\subsection{The informative triads}

Table 3 gives the full set of 16 index-triads that are informative in the sense that each of them implies the full hexad identity. Practically, this means that if a given dataset contains variables in one of the combinations displayed in Table 3 that the entire temporal relationship is available to the researcher.

Note that the 12 possible pairs of intersecting edges on the tetrahedron are, in fact, the informative dyads described in Table 2, whereas the uninformative dyads LP, CT, and $\mathrm{AD}$ are the pairs of opposite edges of the tetrahedron. As discussed, all 12 informative dyads generate one of the four triad identities, but no dyad will generate the hexad identity since a third "independent" time dimension is necessary. Similarly, any quad of indices is sufficient to complete the hexad identity, as at least one of them will not belong to the same face of the tetrahedron, but a triad may be sufficient if its edges do not all belong to the same triad identity.

Table 3: All triads from which the full tetrahedron is derivable (same orientation).

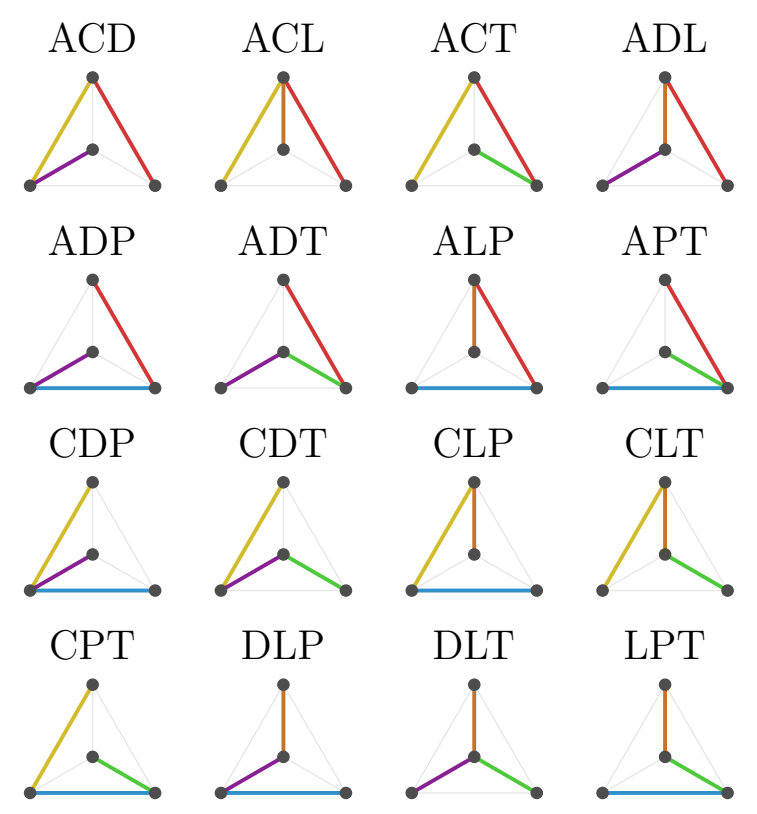

\subsection{The extension of time axes.}

We have said that planes defined by the four triad identities are parallel to the faces of the the above-described tetrahedron. In imagining this three-dimensional relationship, 
we are no longer confined to the extent of the tetrahedron used thus far for orientation. Instead each of its edges extends a certain distance in either direction, and each face of the tetrahedron tessellates to fill its plane. It may therefore help to first consider the extension of each axis (or index). Some indices have a lower bound of zero and an upper bound set by the maximum length of life, $\omega$, while others are boundless. A, T, and L are clearly in the range $[0, \omega] .^{7} \mathrm{P}, \mathrm{C}$, and $\mathrm{D}$ are bounded only by the inception and extinction of our species, but may be thought of as boundless, or benchmarked to our earliest and most recent observations for practicality. ${ }^{8}$ As an abstraction, however, the dimension of calendar time in this model is infinite. Of the four triad identities, only one lacks an unbounded dimension, the TAL. Adding the absent dimension to TAL therefore makes its $3 \mathrm{~d}$ extension boundless. In this way, we may imagine a prism-like construct, where $\mathrm{T}$, A, and L, compose the faces of a triangular cross-section of the prism, which extends infinitely "through" the triangle. We can think of the TAL triangle passing through time, extending the population forward into the future and backward into the past. In this case, the TAL triangle may take either the period or cohort perspective.

\section{Diagram of the hexad identity}

There are different ways to proportion this three dimensional construct, of which we only present the isotropric mapping. In an isotropic projection, the tetrahedron is regular, such that all edges are of the same length, and the units of each of the six represented time measures are therefore equal. In this case, the four triad identities map to temporal planes as tessellations of equilateral triangles, and the four planes are joined together such that each is parallel to a face from the regular tetrahedron. When the plane parallel

\footnotetext{
${ }^{7}$ It's best to imagine some number like 122.45 years, for $\omega$, rather than infinity. This is the longevity record at the time of this writing. Jeanne L. Calment would have had $T=122.45$ at birth, $A=122.45$ at death, and $L=122.45$ for her entire life.

${ }^{8}$ We explain the choice of the word "benchmarked". Say we have a data series that runs from 1751 to 2011, and an upper age interval of $110+$. Then we could say that $\mathrm{P}$ is in the range $[1751,2011]$, but by another reading, $\mathrm{P}$ must range from at least as early as the earliest $\mathrm{C}$ and until at least as late as the latest D. Someone dying at 110 in 1751 had a C of 1640, and an infant born in 2011 that is destined to live to 110 will die in 2121 . In this case a $\mathrm{P}$ that contains the observed population will extend well before and after the observed data series, even more so if we take into account that $\omega>110$.
} 
to each respective face is repeated in equal intervals, we have an isotropic $3 \mathrm{~d}$ space. ${ }^{9}$ Displaying all planes simultaneously creates a very dense and difficult-to-read diagram. We opt to delineate the space using particular planes and intersections.

Figure 6 gives a view of a demographic time diagram that corresponds to the hexad identity, where birth-cohort TAL cross-sectional planes are placed in sequence in a perspective drawing. ${ }^{10}$ The most recent TAL plane, for the year 2000, is placed in the front, whereas past TAL planes are stacked behind it, highlighted in 25-year intervals. The left edge of the frontmost TAL plane is labelled as an axis for thanatological age, although the same tick marks also serve for completed lifespan. The base of this figure is the APC plane, drawn for thanatological age 0. Each of the TAL planes sits atop a single birth cohort line from the familiar APC plane that makes up the base of the figure.

For example, imagine an infant born in the year 2000. Without further information, we only know that this infant is located somewhere on the thanatological age axis of the front TAL plane. If this infant is destined to die in the year 2100, then the vertical position at birth will be at the axis tick for thanatological age 100. This person's entire life stays on the 100 lifespan line (labelled), descending over time towards thanatological age 0. Point A marks the midpoint in life for this individual, at chronological age 50 (red line, labelled), and thanatological age 50 (green line). If another APC plane were drawn through thanatological age 50, we would see that point $\mathrm{A}$ is in the year 2050. Since all individuals born in the year 2000 complete the same age in the same year, we can also recuperate the year for point $\mathrm{A}$ by following the chronological age 50 line (red) down to where it meets the blue line for the year 2050. The lifeline descends downward toward the APC plane for thanatological age 0 at chronological age 100, meeting the year 2100 , which determines the death cohort.

The density and location of imaginary lifelines in this diagram, omitting migration,

\footnotetext{
${ }^{9}$ The isotropic space that results from this framework is known in other disciplines with different nomenclatures. In geometry, this structure is called the tetrahedral-octahedral honeycomb, a variety of space-filling tessellation. In architecture, it is found in the octet truss system. In physics it is called the isotropic vector matrix. Constructs following this geometry exist in nature, in other theoretical settings, and in man-made structures.

${ }^{10}$ The coordinates used to render Figure 6 are isotropic. However, there are no $60^{\circ}$ angles in this figure due to the use of parallax and an indirect viewing angle in this rendering for the sake of increased legibility.
} 
Figure 6: Diagram of the hexad identity, showing a sequence of TAL planes intersecting with a single APC plane.

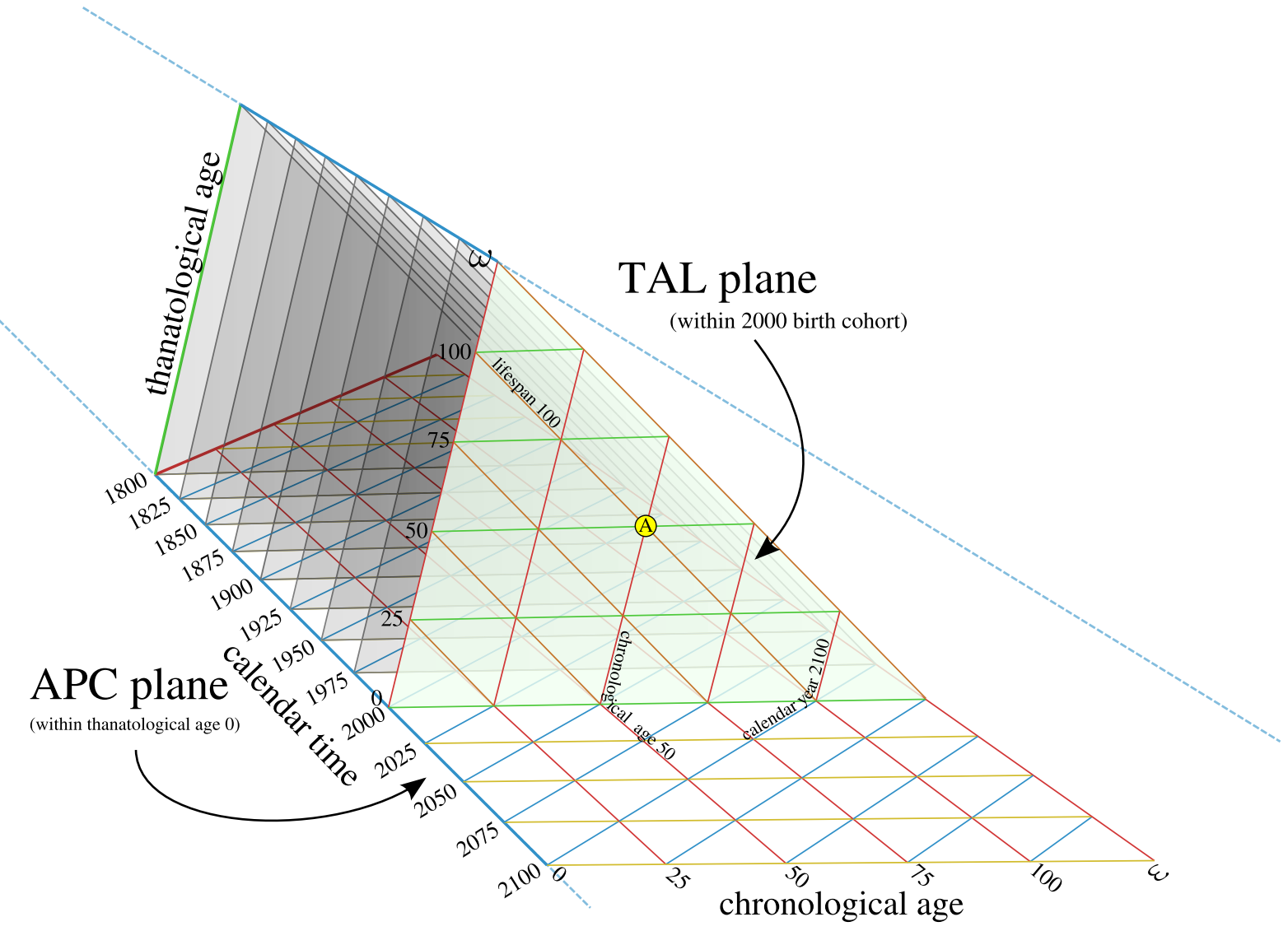

is purely a function of birth cohort size and survival. For extinct cohorts all lifelines can be positioned, but for the 2000 birth cohort this is not yet the case. Most of the front TAL plane is in the future. One may imagine yet another plane intersecting this space - the "present plane", which is identical to the period TAL plane for the present moment. To see how this plane divides the space, imagine that we are in the year 2025, and follow the blue line in the APC base inward 25 years to where it meets the red line for chronological age 25, and follow the red line up the front TAL plane. A single plane cuts through the year 2025 and chronological age 25 from the year 2000 birth cohort. This plane shifts forward or backward in time to meet the present year. In this particular plane, the coordinates $\mathrm{T}, \mathrm{L}$, and $\mathrm{D}$ are uncertain. The period TAL plane $\omega$ years in the past is fully identified, ergo, theoretically the lifespan of each individual in the time of Lexis is knowable.

Figure 6 could have been drawn with TPD or LCD planes highlighted as well, but these 
can still be imagined upon the current rendering. TPD planes transect this space through any given chronological age, for instance. Imagine a wall on the left side of the prism, cutting through chronological age 0 (recall Figure 2). In this case, the thanatological age axis is indicated in the very back of the diagram, calendar time becomes another axis, and death cohort diagonals are not drawn. TPD planes sequence inward from this first plane, always forming cross-sections through chronological age. The LCD plane is to be found by rotating the current prism such that the angle of view is directly down (or up) lifelines, which would then appear as points (recall Figure 4).

Further, the two classes of planes drawn (APC and TAL) could have been drawn and labelled differently. In practice, the diagram of any triad identity may be drawn held constant for any one of its three missing indices with no loss of generality. For example, we have mentioned period $(\mathrm{P})$ and birth cohort $(\mathrm{C})$ TAL planes, but there must also be a third TAL plane that is held constant for death cohort (D). Another example of such multiplicity is found in the birth cohort TAL diagram. In this diagram, lifespan lines can also be interpreted as death cohorts (D), chronological age lines are also period lines $(\mathrm{P})$, and thanatological age $(\mathrm{T})$ is maintained. This means that the birth cohort TAL diagram is also a birth cohort TPD diagram. Multiplicity of this kind can be systematically confirmed, since the $\mathrm{C}$ time measure is not part of TPD. To not overly extend this exposition, and to avoid undue confusion, we neither delineate every possible cross-section nor the possible interpretations of each cross-section. However, in general there are four total ways to cut the space that are parallel to the faces of the hexad identity, and each cut has three possible diagram interpretations.

The essential property of this perspective diagram is that lifelines start and end in parallel, desceding downward and forward in time. A real population of renewing lives, spread over time and over the typical range of human lifespans, will tend to fill the entirety of the prism depicted in Figure 6, and any given point in the prism can be given six demographic time coordinates, of which two or three are redundant. 


\section{Application}

The relationship between the six measures of demographic time is true in the same sense that mathematics is true: Under linear time it is an internally valid set of relationships, and this is self-evident. We have mentioned that the coordinates described here may be useful for the visualization of data, to enable discovery, and to better inform demographic methods. We have not yet mentioned how such developments might arise in practice. We therefore give a schematic overview of our own process of scientific inquiry and reflection that was based this coordinate system, and that would not have arisen without it. This chain of inquiry is meant to demonstrate the usefulness of the present framework, but it is far from an exhaustive application of its potential for other substantive questions, nor is the case study described in complete rigor. Specifically, we reason that projections or comparisons of healthy life expectancy (HLE) are in many cases biased in period prevalence-based models unless one takes into account the thanatological age pattern of prevalence, as well as mortality differences.

There are three steps in our empirical inquiry. The first step is to visualize variables on health outcomes using our framework. The second step is to assess the primary time measures over which health outcomes appear to vary. Under the assumption that these patterns of temporal variation are empirically regular, we proceed to develop a method of standardizing health expectancy calculations for morbidity conditions whose prevalence is more closely related to thanatological age. Finally, one can reason that period estimates of health expectancies for certain health conditions are biased when mortality has been or will-be changing, and comparisons of HLE between populations with different mortality are also biased. We conclude that comparisons of health expectancies might be biased in ways not previously documented.

Let us take the example of self-reported health ( $\mathrm{SRH})$. This variable is available from many different survey sources, and it is familiar to many researchers. There are many known pitfalls to this particular variable that already make it difficult to compare between sexes, over time, or between populations, and these do not concern us. We use this variable as an example because of the clear pattern of variation that it shows, which 
we use to make our more general point.

Figure 7 displays a series of TAL surface plots, each referring to a different quinquennial birth cohort (1905-1909, etc). The data come from the RAND version of the US Health and Retirement Study (RAND HRS Data, Version M 2013). Since this survey has multiple observations of individuals, as well as a mortality follow-up, we have each of the six time measures for each observation. Further methodological details are given by Riffe, T. et al. (2015). The TAL surfaces for each successive birth cohort are shifted by five years because the observation window available is from 1992 to 2011 for each cohort.

Figure 7: Prevalence of males self-reporting poor health by chronological and thanatological age, by quinquennial birth cohorts, 1905-1925. (HRS)

(a) 1905

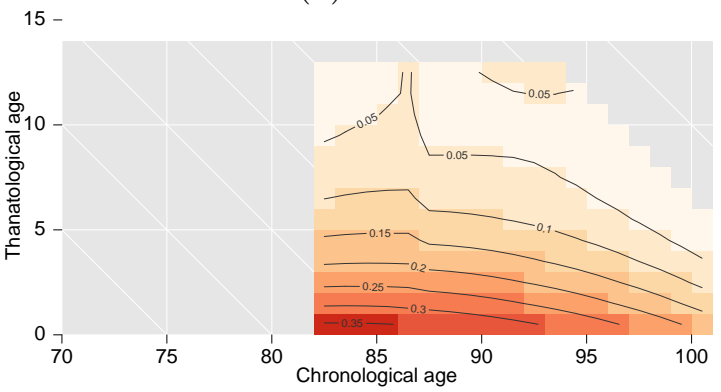

(c) 1915

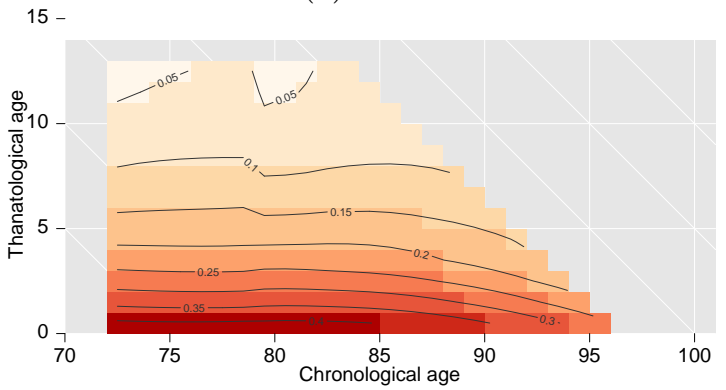

(e) 1925

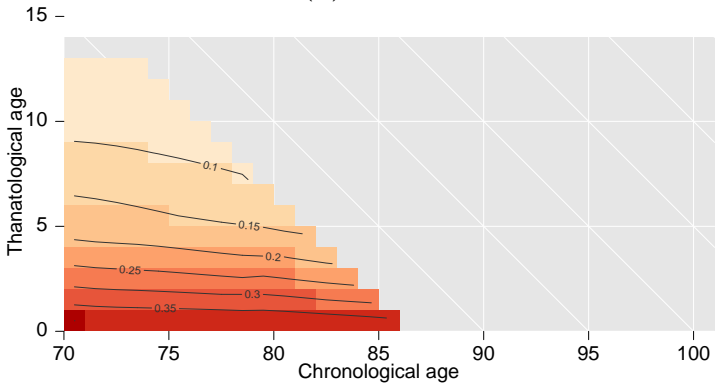

(b) 1910

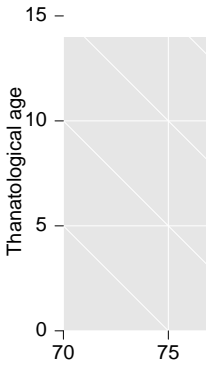

$15-$

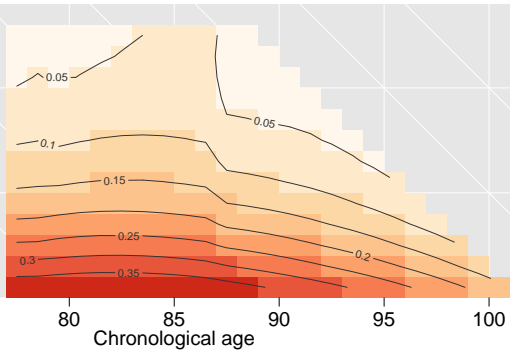

(d) 1920
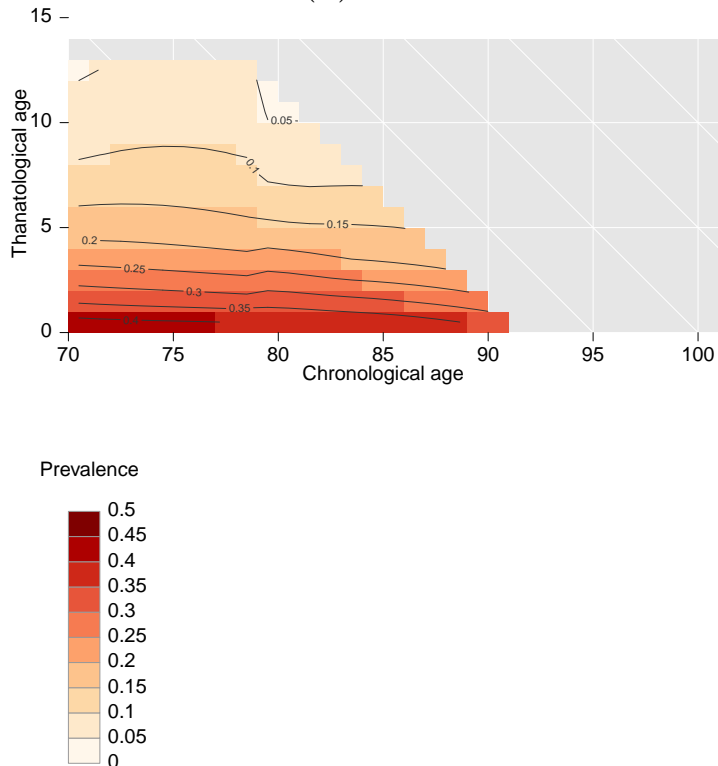

The $x$-axis of these surface plots is chronological age, while the $y$-axis is remaining years of life. Contour lines in the surfaces indicate the primary direction of variation, in this case over thanatological age. Downward diagonals indicate lifespans, which the reader may also think of as very specific birth-death cohorts. These are the diagonals along which 
lifelines may be imagined, as suggested in Figures 3 and 6. For each of these birth-death cohorts we have a prevalence trajectory - empirical examples of the lifeline morbidity trajectories often conceptually diagrammed in the literature on morbidity compression (e.g., Fries 2005). In each surface the primary direction of variation is along thanatological age, and not chronological age. The prevalence for those with $t$ remaining years of life is similar in these data, irrespective of chronological age, birth cohort, or ultimate lifespan.

When one looks at a chronological age pattern of SRH, as measured here (the Sullivan curve, (Sullivan 1971)), one sees an increasing tendency over age. However, such an increasing line is a marginal ruse, due to an interaction between the distribution of lifespans and the relatively fixed underlying pattern of morbidity seen in Figure 7 . These surfaces can indeed be tidily summarized with a single line, but it is a line over the thanatological age margin rather than over chronological age.

Since the patterns for each of these cohorts can be presumed to be the same, any shifting in the distribution of lifespan ought not produce a change in the expected years of poor health for a given lifespan. Further, the life years spent in poor health should also be approximately the same "on average", even if the underlying mortality patterns shift. If morbidity change is a pure function of thanatological age, an increase in life expectancy should increase healthy life expectancy by the same amount. This is not the prediction when we base analyses on the chronological age pattern of self-reported health. An underlying morbidity pattern this stable would predict improvements in the marginal chronological age pattern of self-reported health if the lifespan distribution were to shift to higher ages. This bias in the current status quo of morbidity measurement and prediction leads to pessimistic morbidity scenarios when mortality improvements are foreseen, and it undermines health expectancy comparisons between groups with different mortality (Van Raalte \& Riffe 2016). Cohort health expectancies are in either case unbiased, but these are also not common.

Using the data from our example surfaces, we can calculate an average prevalence trajectory with the approach to death and calculate some basic results that support our case. Let us take the population of US males aged 60 and older, and assume that the 
trajectory derived from the Figure 7 surfaces is valid for them. If we apply this trajectory to the synthetic stationary population of each year from 1980 and 2010 (HMD 2016), we can calculate the resulting healthy and unhealthy life expectancies, and compare these with the expectancies that we would have projected assuming the 1980 Sullivan curve. Total remaining life expectancy at age 60 increased 4.3 years from 17.4 to 21.7 years from 1980 to 2010. Assuming the thanatological trajectory of morbidity, we calculate healthy life expectancies of 15.7 and 19.9, respectively, an increase of 4.2 years. Unhealthy life expectancy in this scenario increased just 0.1 years. Had we used the Sullivan curve from 1980 to calculate the 2010 values, we would have predicted an increase of 0.7 years in unhealthy life expectancy, or $39 \%$ versus the $4 \%$ "observed" in this exercise.

This is a large difference in projected morbidity, and it is based on a relatively minor tweak to standard methodology, itself inspired by viewing data under the conditions enabled by this temporal framework and adjusting standard demographic methods to capture the direction of temporal variation in data. There is a wide variety of prevalence patterns when viewed in this way (Riffe, T. et al. 2015, Wolf et al. 2015), and much empirical and methodological work is still required to verify that these findings are representative and to understand the consequences for the standard ways of comparing and projecting HLE.

Our objective in this application has been to demonstrate how viewing data under the rigorous conditions enabled by the time-framework we propose can lead to new scientific understandings of processes over the life course. Applications for other aspects of the lifecourse, particular stages of the lifecourse, non-human species on all time scales, populations of inanimate items on all time scales, and myriad other substantive areas may gain new insight by applying the relationships contained in the demographic time identity or its consituent triad identities. 


\section{Related concepts}

A simultaneous juxtaposition of the six demographic time measures has never appeared in the literature. However, Lexis (1875) describes an analogous hexad relationship between, birth cohort, age at marriage, age at separation (death or widowhood), marriage cohort, separation cohort, and duration of marriage. The hexad relationship between these six time measures can also be verified on a tetrahedron graph. Lexis also describes crosssections in the same sense (Schnitte) that we do, denoting two-dimensional diagrams, with values of the orthogonal dimension held constant. This identity is coherent, but it does not find perfect analogy in our proposed framework, in part because each lifeline in the Lexis hexad identity has up to three possible points. The period dimension is not explicit, but rather nested within three varieties of cohort. Clearly with age, period, and total duration, remaining time should also be identifiable, but this is not included in Lexis' hexad identity.

Our hexad identity is designed to contain and identify any point on the lifecourse of individuals in populations with heterogeneous lifespans. As such it fully describes a population of single state durations. We liken Lexis' hexad identity to something similar to a two state APC, albeit without explict period. Lexis' marriage hexad identity is also illustrated with complex perspective line drawings (see Lexis' Figures 5 and 6). These figures (based on cubes rather than tetrahedra-octahedra) and the portion of the text that discusses these relationships has unfortunately been largely overlooked in posterior literature, which has focused much more on the APC plane. We do not claim that the concepts in Lexis' hexad identity are not transportable, but perhaps the specificity of this identity has made it unwieldy in practice, or difficult to conform with data. Adding an additional state to the identity we propose would also greatly expand its complexity.

Lifelines represented in our hexad diagram are also reminiscent of the Lexis pencil notion from Francis \& Fuller (1996). Lexis pencils are a method of representing limited numbers of multistate lifelines in three dimensional space, where lifelines ascend diagonally from age zero. This visualization system could be constrained to match the one we present if the $z$ axis were set to remaining years of life and lifeline trajectories were 
realigned to descend toward zero remaining years of life (or some rotation thereof), and sorted by length of life.

\section{Discussion}

In isolation, each of the four triad identities is intuitive. The TPD diagram is similar to APC, but it is aligned to time of death rather than time of birth. The TAL diagram presents a clear way to classify events over the life course of a cohort. Finally, the LCD diagram can be used to structure quantities that vary over time and by length of life. Joined together, the relationship between all six time measures is more complex than any of the triad identities, but it condenses into a simple geometric representation that can be easily derived by ascribing the various time measures to the six edges of a tetrahedron. An understanding of how the six time measures relate is key to understanding the temporal structure of demographic processes, which itself underlies the comparability of demographic measures.

The contemporary practice of (macro) demography is based on the premise that vital rates, and other kinds of rates over the lifecourse, are the truest measure of demographic forces. Rates are paramount because they tend to vary in empirically regular ways over the life course. The scalings and movements of primary vital rates fall within a limited range for humans. For this reason, many of the methods of demography are developed to estimate rates, independent of population composition, or to partition crude magnitudes into the effects of population age structure and pure vital rates. Controlling for age like this is in a more general sense controlling for temporal variation in stocks. To the extent that regular temporal variation relates to the end of life, or the length of life, common age-standardization does not fully account for such structure.

The techniques used to age-standardize mortality and fertility estimation are at times applied to other kinds of transitions over the life course. For example, one may estimate an age pattern to some degenerative disease, or the ability to carry out some common activities of daily life. However, much of the regular temporal variation for the preva- 
lence of such conditions is by time-to-death or lifespan, rather than by chronological age. Apparent chronological age patterns in such cases are artifactual and do not represent the same kind of intrinsic meaning as does the age pattern of mortality. Further kinds of temporal standardization must be developed in order to measure and understand the natural patterns of such conditions over the lifecourse. The measurement of such conditions may benefit from consideration of the framework presented in this paper. To this end, Table 3 provides all combinations of information that are sufficient to derive the full set of six time measures. Panel surveys with mortality follow-ups already provide the requisite information, as do linkable registers that include items such as health measures or proxies and relevant dates of birth, observation, and death. Other kinds of populations, such as animals or items, may have quite different data-gathering mechanisms.

An effective way to detect patterns in temporal variation is via data visualization. The coordinate system proposed in this paper is conceived as one adequate to capture such variation, and we suggest its use for visualizing data, probably via small multiples of successive time slices parallel to any of the four triad identities, similar to that shown in Figure 7. Such visualization strategies at this time are exploratory, and this is a technique that may benefit from further refinement. Further, a cross-section through the demographic time-space need not be parallel to one of the four identity-planes.

Mortality determines three of the dimensions of demographic time, and it therefore makes little sense to model mortality using all six time measures. Rather, mortality determines the placement of lifelines in the three-dimensional space. Death itself is only representable with the endpoints of lifelines, ergo the APC plane through thanatological age zero. This full coordinate system instead defines and contains the space through which life is lived; any of the six measures may be pertinent in the case of conditions and states that vary over and within the lifecourse. An obvious application for the present model, given data commonly (and publicly) available at this time, are late-life health conditions, although there may be other substantive areas of application.

Furthermore, we believe in the pedagogical value of the framework introduced in this paper. We hope that the present inquiry will be useful as a teaching instrument 
in the same way as APC diagrams have formed a part of basic demographic education. The relationship between the six dimensions of demographic time helps situate the APC paradigm in a broader framework. Just as scientific discovery in general depends partly on the development of finer optics and instrumentation, we hope that the framework we describe will prove an instrument to enable new discoveries in formal, and empirical demography, as well as other diverse fields of investigation.

\section{References}

Caselli, G., Vallin, J., \& Wunsch, G. (2006). Demography: Analysis and synthesis, four volume set: A treatise in population (Vol. 1). Academic Press.

Francis, B., \& Fuller, M. (1996). Visualization of event histories. Journal of the Royal Statistical Society. Series A (Statistics in Society), 159(2), 301-308.

Fries, J. F. (2005). Frailty, heart disease, and stroke: the compression of morbidity paradigm. American journal of preventive medicine, 29(5), 164-168.

Human Mortality Database. (n.d.). University of California, Berkeley (USA) and Max Planck Institute for Demographic Research (Germany). (Available at www.mortality.org or www.humanmortality.de (data downloaded on July 10, 2014).)

Keiding, N. (2011). Age-period-cohort analysis in the 1870s: Diagrams, stereograms, and the basic differential equation. Canadian Journal of Statistics, 39(3), 405-420.

Knapp, G. F. (1868). Über die Ermittlung der Sterblichkeit aus den Aufzeichnungen der Bevölkerungs-Statistik. JC Hinrich.

Lexis, W. (1875). Einleitung in die Theorie der Bevölkerungsstatistik. KJ Trübner.

Müller, H.-G., Wang, J.-L., Carey, J. R., Caswell-Chen, E. P., Chen, C., Papadopoulos, N., \& Yao, F. (2004). Demographic window to aging in the wild: constructing life tables and estimating survival functions from marked individuals of unknown age. Aging Cell, $3(3), 125-131$. 
Müller, H.-G., Wang, J.-L., Yu, W., Delaigle, A., \& Carey, J. R. (2007). Survival and aging in the wild via residual demography. Theoretical population biology, 72(4), 513522.

Perozzo, L. (1880). Della rappresentazione graphica di una collettivita di individui nella successione del tempo. Annali di Statistica, 12, 1-16.

Pressat, R. (1961). L'analyse démographique: méthodes, résultats, applications. Population (french edition), 16(3), 505-508.

van Raalte, A., \& Riffe, T. (2016). Accounting for temporal variation in morbidity. Presented at the Annual Meeting of the Population Association of America.

RAND HRS Data, Version M. (2013, September). Santa Monica, CA. (Produced by the RAND Center for the Study of Aging, with funding from the National Institute on Aging and the Social Security Administration.)

Riffe, T. (2015). The force of mortality by life lived is the force of increment by life left in stationary populations. Demographic Research, 32(29), 827-834.

Riffe, T., Chung, P. H., Spijker, J., \& MacInnes, J. (2015). Time-to-death patterns in markers of age and dependency. MPIDR Working Papers, WP-2015(3), 25.

Sanderson, W., \& Scherbov, S. (2007). A new perspective on population aging. Demographic research, 16(2), 27-58.

Sullivan, D. (1971). A single index of mortality and morbidity. HSMHA Health Reports, $86,347-354$.

Vandeschrick, C. (2001). The lexis diagram, a misnomer. Demographic Research, 4(3), $97-124$.

Vaupel, J. W. (2009). Life lived and left: Carey's equality. Demographic Research, 20(3), $7-10$. 
Villavicencio, F., Jordà, J. P., \& Pujadas-Mora, J. M. (2015). Reconstructing lifespans through historical marriage records of barcelona from the sixteenth and seventeenth centuries. In Population reconstruction (pp. 199-216). Springer.

Villavicencio, F., \& Riffe, T. (2016). Symmetries between life lived and left in finite stationary populations. Demographic Research, 35(14), 381-398.

Wilmoth, J. R., Andreev, K., Jdanov, D., Glei, D. A., Boe, C., Bubenheim, M., ... Vachon, P. (2007). Methods protocol for the human mortality database (Tech. Rep.). University of California, Berkeley, and Max Planck Institute for Demographic Research, Rostock. Retrieved from http://mortality.org[version31/05/2007]

Wolf, D. A., Freedman, V. A., Ondrich, J. I., Seplaki, C. L., \& Spillman, B. C. (2015). Disability trajectories at the end of life: A countdown model. The Journals of Gerontology Series B: Psychological Sciences and Social Sciences, 70(5), 745-752.

Zeuner, G. (1869). Abhandlungen aus der mathematischen Statistik. Verlag von Arthur Felix. 